\title{
What is the Optimal Treatment for Metastatic Colorectal Cancer? Controversial Points
}

\section{Esther Uña Cidón}

Clinical Oncology Department, Faculty of Medicine, Clinical University Hospital of Valladolid, Spain

\begin{abstract}
Colorectal cancer (CRC) is the second leading cause of death from cancer in the developed countries. Although great efforts have been made to achieve an early diagnosis, a large number of cases will present metastases. The natural history of metastatic $(\mathrm{m}) \mathrm{CRC}$ has dramatically evolved in the recent years thanks to the introduction of modern chemotherapy and molecular therapies. With these agents the response rate has increased to $50 \%$ and survival has been improved not only progression free survival (PFS), which has reached 12 months, but also overall survival (OS) which is longer than 2 years.
\end{abstract}

Despite this progress many questions remain to be answered, mainly those related to the sequential regimens, drug rotation, alternant or intermitent schedules, optimal duration of chemotherapy, the role of maintenance chemotherapy and the role of doublets or triplets.

The optimal duration of chemotherapy is very important because it has a direct influence on the patient quality of life, survival and costs. There are several studies addressing this topic and the alternatives we have, such as "stop and go", intermittent strategies or maintenance of only several agents and these studies reinforce the frequent behaviour of the oncologists to stop the treatment when the patient has obtained the maximum response. But there are some methodological problems in the analyzed trials which have determined that not all the professionals agree with this proposal.

With this context it is essential to perform well designed clinical trials incorporating new drugs and addressing these questions. This article tries to review briefly all these controversial points.

Keywords: Colorectal Cancer; Metastases; Chemotherapy; Biologics

\section{Introduction}

CRC is the fourth most common cancer around the world with an increasing incidence and is still the second leading cause of cancer related death [1].

In spite of current efforts in improving screening programs, 20\% of patients are diagnosed once their tumor has metastasized [2] and ultimately metastases develop in as many as $50-60 \%$ of patients with CRC, often in the liver [2]. This subgroup of patients has a much worse outcome, with 5-year survival of approximately $10 \%$. Most of these patients cannot be cured, although a subset with hepatic and/or lung metastases might be potentially curable with surgery. For the rest, treatment's goal is palliative and usually consists of systemic therapy [2].

Long-term survival is infrequent once metastatic disease has appeared and is limited to a very small proportion of patients that can undergo metastasectomy. Over the last 10-15 years the integration of new drugs such as oxaliplatin and irinotecan has allowed us to improve the overall survival (OS) of mCRC patients.

In fact the median OS for patients with mCRC has doubled. This was accomplished mainly due to the introduction of newer chemotherapeutic drugs and regimens, including the use of biologics or targeted agents. The median overall survival (OS) improved from 10 12 months in patients treated with 5 -fluororuracil (5-FU)/leucovorin (LV) $[3,4]$ to $20-21$ months reported in recent clinical trials using a 3 drug combination [5].

Nowadays there are seven drugs approved by the FDA for the treatment of $\mathrm{mCRC}$ which are frequently used in combination. On average, patients undergo two to three lines of systemic treatment, thus the current therapeutic algorithm is much more complex than a decade ago. According to the last update, the National Comprehensive Cancer Network (NCCN) guidelines consider 12 different drug combinations as possible options for first line treatment in patients with Mcrc [6] Therefore it is relevant to fully understand the efficacy and different toxicity profiles of these agents in order to better tailor our therapies to each individual patient.

\section{Chemotherapy Versus Best Supportive Care}

The median OS for patients with unresectable $\mathrm{mCRC}$ who receive the best supportive care alone is approximately five to six months. Systemic chemotherapy compared to the best supportive care get an advantage in progression-free survival (PFS) and OS [7].

Although no randomised trial has compared modern regimens containing oxaliplatin or irinotecan to best supportive care alone, with these modern drugs the median survival duration across different studies consistently approach to 20-24 months [5].

*Corresponding author: Esther Uña Cidón, MD, PhD, Professor, Clinical Oncology Department, Clinical University Hospital and Faculty of Medicine, C/ Ramon y Caja s/n, 47005 Valladolid, Spain, Tel: 34 678938050; E-mail: aunacid@hotmail.com

Received October 03, 2011; Accepted November 14, 2011; Published November 16, 2011

Citation: Cidón EU (2011) What is the Optimal Treatment for Metastatic Colorecta Cancer? Controversial Points. J Cancer Sci Ther S4:003. doi:10.4172/1948-5956. S4-003

Copyright: ( 2011 Cidón EU. This is an open-access article distributed under the terms of the Creative Commons Attribution License, which permits unrestricted use, distribution, and reproduction in any medium, provided the original author and source are credited. 


\section{Timing to Institute Chemotherapy}

Many patients with $\mathrm{mCRC}$ are asymptomatic and the value of early chemotherapy vs delay the treatment until symptoms appear is controversial.

The appropriate timing of the use of "expectant" chemotherapy has been the subject of debate, especially when the potential toxicity of treatment is taken into account.

There are a few relevant studies addressing directly this question. The study by the Nordic Gastrointestinal Tumor Adjuvant Therapy Group [8] randomised 183 patients with advanced but asymptomatic CRC. The results showed an improvement in median survival, symptoms free interval and PFS but it had several methodological issues (chemotherapy was not standardized, only 57\% of patients received chemotherapy in the expectant arm, no formal Quality of Life (QoL) analyses were published...) preventing a clear and definitive conclusion.

On the other hand the meta-analysis by Ackland et al. [9] showed a two months benefit in median OS with early treatment but although clinically relevant it was not statistically significant. There were not any differences in QoL, so authors concluded that early treatment of asymptomatic patients with $\mathrm{mCRC}$ did not provide any benefit compared to withholding treatment until symptoms appeared.

\section{Sequential Versus Combination Therapy}

Capecitabine is an oral pro-drug of 5-FU mainly metabolized within tumor cells and liver to 5-FU $[10,11]$. Two randomized clinical trials $[12,13]$ have demonstrated noninferiority of this drug to both infusional and bolus 5-FU/LV in mCRC patients. After that FDA approved single-agent capecitabine in the United States in 2002 for first-line therapy where combination therapy was not recommended.

On the other hand combination of capecitabine and oxaliplatin (CapeOx) has been demonstrated not to be inferior to FOLFOX (Ox and 5-FU) [14-17]. However, the combination of capecitabine and irinotecan (CAPIRI) resulted in intolerable toxicities and a shorter PFS in two randomized trials, effectively discouraging use of this regimen, especially in the United States where capecitabine is less well tolerated at standard doses [15,18-20].

There are several studies evaluating the efficacy of combination therapy versus single-agent sequential therapy.

CAIRO study randomized previously untreated patients (N 803 patients) with advanced CRC to either sequential or combination treatment [21]. In the sequential arm, patients received full-dose capecitabine followed by second-line single agent irinotecan at full dose $\left(350 \mathrm{mg} / \mathrm{m}^{2}\right.$ every 3 weeks) on progression. Third line therapy was a combination of capecitabine and oxaliplatin (just 36\% of the original patients remained)

The results showed that combination did not prolong OS (17.4 versus 16.3 months; $\mathrm{p} 0.33$ ) versus sequential therapy although PFS was significantly superior in the combination arm (7.8 versus 5.8 months), and also overall response rate ( $41 \%$ versus $20 \%)$.

The study by Seymour and colleagues (Medical Research Council (MRC) FOCUS) randomized patients to 5 treatment strategies across 3 arms [22]:
- Arm A: 5-FU until progression, then switch to irinotecan

- Arm B (Ir): 5-FU until progression, then add irinotecan

- $\quad \operatorname{Arm~B~(Ox):~5-FU~until~progression,~then~add~oxaliplatin~}$

- Arm C (Ir): FOLFIRI until progression

- Arm C (Ox): FOLFOX until progression

The results showed that OS for arm A (control) was 13.9 months, arm B [Ir] 15.0 months, and for those instead adding oxaliplatin (arm B [Ox]), the overall survival was 15.2 months. As expected, no significant difference was seen in arm B versus arm A regardless of whether irinotecan or oxaliplatin was added to 5-FU on failure. In arm C, FOLFIRI until failure was associated with statistically significant OS improvement (16.7 months; $P=.01$ ) whereas FOLFOX (15.4 months) until failure was not.

This additional option of either an oral or intravenous fluoropyrimidine in combination with oxaliplatin, or as a single-agent, allows for further individualization of care of CRC patients, depending on patient performance status and if tumor response is the preferred outcome.

These both studies support an alternative to aggressive combination of chemotherapy in non-curative setting.

Indeed Grothey et al. [23] conducted a retrospective analysis of 21 arms from 11 phase III trials that involved slightly fewer than 6000 patients with advanced CRC. None of these trials included biologic agents. The investigators determined that treatment with all 3 active agents (5-FU, irinotecan, and oxaliplatin) was significantly correlated with OS, which suggests that combination therapy should remain the standard of care for first-line treatment of patients with advanced CRC. However, a multivariate analysis revealed that only exposure to 3 drugs $(\mathrm{p}=0.0001)$, and not use of first-line doublets, was associated with an improvement in OS.

\section{Duration of Therapy}

The duration of therapy in mCRC patients is crucial because it has a direct influence on the patients' QoL, toxicity, cost and potentially with survival. But this point is still a controversial one. The classical way has been considered treatment until progression or unacceptable toxicity. However, taking into account that more than $80 \%$ of patients will have a treatment just with palliative meaning it seems to be relevant to maintain QoL without compromising their survival.

At this point in palliative setting we could consider two subgroups of patients: those who present tumor-related symptoms and those with only mild symptoms. Both subgroups have different needs. The first group needs a treatment which provides tumor shrinkage in order to get a rapid response for the control of the symptoms. But the second needs a different strategy named "continuum of care" to provide those patients with better survival and also with QoL.

Different strategies have been used to answer the question about the optimal duration of chemotherapy in these patients:

1. "Stop and go" strategy which means stopping all agents after a prefixed number of cycles and then to restart chemotherapy again on progression. 
2. Intermittent administration of chemotherapy or on-off strategy.

3. Maintenance chemotherapy.

\section{Stop and go}

MRCC trial, OPTIMOX-2 and MRC-COIN study have addressed this issue.

OPTIMOX-2 compared chemotherapy discontinuation with maintenance therapy with leucovorin and 5FU after six cycles of FOLFOX [24]. This trial was planned as a phase III but converted to a smaller phase II trial after bevacizumab was introduced, so the survival data should be interpreted with caution. Duration of disease control was the main end-point and was inferior in the discontinuation arm (9.2 versus 13.1 months). PFS was also inferior. Authors concluded that the arm with planned chemotherapy breaks had inferior results suggesting that this is not the best therapeutic approach. Regardless these result it can be an option to individualize patient treatment based on patient preferences, particularly if there is a strong early response that is durable beyond 6 months.

MRCC trial with more than 350 patients from 42 UK centers concluded that although PFS and OS were slightly superior in the continuous arm, the difference was not statistically significant and toxicity was lower in the patients with the intermittent treatment. Although these results do not provide a definitive evidence of the benefit for the continuous treatment until progressive disease is present, this study did not include modern drugs such as oxaliplatin or irinotecan.

The MRC-COIN trial (CapeOx or FOLFOX with or without cetuximab) had an intermittent chemotherapy arm that showed a $9 \%$ increase in relative risk of death with a significant HR of 1.084 , when compared to the two continuous chemotherapy arms. Authors concluded that a priori specified non-inferiority cannot be confirmed, but it can reliably exclude a detriment of larger than 2.3 months in median OS with intermittent chemotherapy [25].

\section{Intermittent (on-off strategy)}

The Italian GISCAD group [26] compared the administration of the continuous FOLFIRI regimen versus the intermittent FOLFIRI regimen (alternating 2 months on and 2 months off). The results showed the same OS (17.6 versus 16.9 months) and PFS (7.3 versus 8.8 months) in both arms and a reduction of toxicity and costs.

\section{Maintenance strategy}

MACRO-TTD trial [27] randomized 480 first-line patients to either CapeOx-bevacizumab until progression (N 239) or CapeOxbevacizumab for six cycles then single-agent bevacizumab (N 241). There were not statistically significant differences in response rates, PFS, or OS between the groups and toxicities grade 3 and 4 were inferior in the maintenance arm concerning hand-foot syndrome, asthenia and neuropathy.

Nevertheless, it failed to meet its non-inferiority PFS primary endpoint (11 versus 10.3 months, p0.59; HR 1.07; 95\% confidence interval [CI], 0.84-1.36). According to these results authors concluded that maintenance therapy with single agent bevacizumab is an appropriate option following the induction with CapeOx-bevacizumab in patients with $\mathrm{mCRC}$.
The CONcePT study compared the administration of intermittent versus continuous oxaliplatin in the FOLFOX plus bevacizumab regimen. Time to treatment failure and PFS were inferior in the continuous administration. In this way monochemotherapy with bevacizumab is a feasible option [28].

OPTIMOX-1 trial compared FOLFOX given until disease progression with FOLFOX7 given for 12 weeks, with planned interruption of the oxaliplatin and continued 5-FU/LV alone as maintenance, and then a planned re-introduction of oxaliplatin at 6 months or at disease progression, whichever came first [29].

There were no differences in response rates, PFS, or OS. Authors concluded that Oxaliplatin could be stopped without compromising the results (stop and go strategy). Moreover, neurotoxicity was significantly reduced with planned interruption of oxaliplatin treatment.

The results of these trials evaluating optimal therapy duration provide more flexibility and personalization of mCRC care. In fact, these studies reinforcing the behavior of the majority of oncologists to stop the treatment when the patient has shown the maximum response.

\section{Sequence of Therapy}

The optimal sequence of therapy for mCRC is an issue which has been addressed in randomized trials $[21,22,30]$. Tournigand et al. [30] evaluated FOLFIRI followed by FOLFOX or the reverse sequence. They found no significant differences in OS or first-line response rates between the two sequences. The toxicity profiles were significantly different: FOLFIRI caused more grade 3 and 4 mucositis, nausea, vomiting, and diarrhea, while neutropenia and neurotoxicity were more common with FOLFOX.

These different toxicity profiles and the ability to choose between these two relatively equivalent regimens provide another alternative based on patient comorbidities and preferences.

\section{Novel Biologic Therapeutics}

\section{Antiangiogenics: Bevacizumab}

Bevacizumab (Avastin ${ }^{\star}$, Genentech, South San Francisco, CA) [31] is a humanized monoclonal antibody that targets the vascularendothelial growth factor (VEGF), binds it and prevents the interaction of VEGF with its receptors (Flt-1 and KDR) on the surface of endothelial cells. VEGF is an angiogenic growth factor that regulates vascular proliferation and permeability and inhibits apoptosis of new blood vessels. VEGF expression is increased in CRC and when it is bound to bevacizumab, it cannot stimulate the growth of blood vessels, avoiding tumors blood and subsequently oxygen and other nutrients needed for growth.

It is administered as an intravenous infusion without necessity of dose modification and indicated for use as part of the combination with chemotherapy.

The most common adverse effects are asthenia, diarrhea, hypertension, headaches, stomatitis or leucopenia, and serious complications are gastrointestinal perforation, bleeding, nephritic syndrome, thromboembolic events and impaired wound healing. This last complication led to not administer this drug for at least 4 weeks prior and after surgical procedures [31].

Bevacizumab has been the the first biologic agent approved by the US FDA as a component of upfront therapy in 2004. 
This decision was based on data from the study by Hurwitz et al. [32], in which 813 patients with mCRC were randomized to IFL + bevacizumab versus IFL alone. The results showed statistically significant superiority in response rate $(44.8 \%$ versus $34.8 \%$ ( $\mathrm{p}=$ $0.004))$, PFS (10.6 versus 6.2 months $(\mathrm{p}=0.001))$ and OS $(20.3$ versus 15.6 months $(\mathrm{p}=0.001))$.

The N016966 trial evaluated the addition of bevacizumab in the front-line setting with either FOLFOX or CapeOx in a placebocontrolled $2 \times 2$ design. Bevacizumab improved PFS, but response rates were not improved, and the differences in OS were not statistically significant (p 0.077) [33].

Since FOLFIRI-bevacizumab and FOLFOX/CapeOx-bevacizumab have not been compared directly in the first-line setting, either is currently considered acceptable. In these cases patient preference and adverse effects profile have contributed to decision-making.

The Eastern Cooperative Oncology Group (ECOG) conducted the E3200 trial, in which patients who progressed on a fluoropyrimidine and irinotecan based therapy were randomized to either FOLFOX 4 plus bevacizumab, FOLFOX 4 or bevacizumab alone. This trial showed response rates of $22.7 \%, 8.6 \%$ and $3.3 \%$ (p 0.0001) respectively. PFS and $\mathrm{OS}$ in the FOLFOX plus bevacizumab group were superior to FOLFOX alone (7.3 versus 4.7 months; 12.9 versus 10.2 months; $p$ 0.001). Bevacizumab alone showed only modest activity [34].

Due to IFL was replaced with FOLFOX and FOLFIRI, these regimens plus bevacizumab (FOLFOX-bevacizumab, FOLFIRIbevacizumab) were widely adopted for first-line use in the United States.

The continued use of bevacizumab in the second line setting beyond progression was addressed in the BRiTE study [35]. This prospective, observational study showed that patients who received bevacizumab beyond progression ( $\mathrm{N}$ 642) had OS of 31.8 months compared to 19.9 months for those who did not (N 531), suggesting that this procedure might be beneficial without apparent increase of bevacizumab-related adverse effects.

The prospective randomized phase III trial iBET [36] (Intergroup Bevacizumab Continuation Trial) Southwest Oncology Group (SWOG) 0600, comparing irinotecan-cetuximab and bevacizumab to irinotecan-cetuximab alone, and the SPIRITT (Second-Line Panitumumab Irinotecan Treatment Trial) phase II trial, in which $[37,38]$.

KRAS wild type patients receive second-line FOLFIRI and are randomized to either panitumumab or bevacizumab are trying to answer this question.

Globally, bevacizumab adds strong benefit in OS and PFS to all regimens in first line and the positive effect of its addition on median PFS and OS is reproducible across different studies. When evaluated in real clinical practice, bevacizumab leads to long PFS when combined with chemotherapy and community-based studies are also supporting its effectiveness.

There exists a controversy about the duration of therapy with bevacizumab. The exploratory analyses by Mass et al has shown that treatment until progression is supported by clinical benefit in OS in responding and non-responding patients. In fact, regardless of response, patients treated with bevacizumab received a significant OS benefit compared to controls, for patients with stable disease for at least 12 weeks as 'best outcome', bevacizumab-treated patients experienced a significant decrease in risk of progression.

This exploratory analysis suggested that objective response may not be an appropriate criterion for stopping treatment being progressive disease the most appropriate criterion.

Moreover, stopping bevacizumab before progressive disease impacts negatively in PFS.

\section{Anti-EGFR (Human Epidermal Growth Factor Receptor): Cetuximab and Panitumumab}

EGFR is overexpressed in about $70 \%$ of patients with mCRC and has been considered a poor prognostic factor. Cetuximab and panitumumab are monoclonal antibodies (chimeric human-mouse, and fully human, respectively) that block the ligand-binding site of the EGFR. As a consequence they inhibit the transduction of this signal intracellularly.

Cetuximab (Erbitux ${ }^{\oplus}$, Eli Lilly, Indianapolis, IN) binds specifically to the extracellular domain of the EGFR on both normal and tumor cells, and competitively inhibits the binding of EGF and other ligands, resulting in inhibition of cell growth, induction of apoptosis, and decreased matrix metalloproteinase and VEGF production.

Cetuximab can also mediate antibody-dependent cellular cytotoxicity against certain human tumor types. It is administered as an intravenous infusion at $400 \mathrm{mg} / \mathrm{m} 2$ (initial dose) followed by a weekly dose of $250 \mathrm{mg} / \mathrm{m} 2$, given either as single agent or in combination with FOLFOX, FOLFIRI or irinotecan. The most common adverse effects are acneiform rash, fatigue, dyspnea, diarrhea and nausea [39].

FDA approved it in 2004 for use in patients who progressed on irinotecan based therapy. The decision was based on a trial which randomized 329 patients to irinotecan plus cetuximab versus cetuximab alone ( $>75 \%$ of the patients had progressed to 2 or more regimens of chemotherapy). If patients progressed on cetuximab they were able to crossover to the doublet. This study showed superiority of the combination in response rate $(22.9 \%$ versus $10.8 \%, \mathrm{p} 0.007)$ and PFS (4.1 months vs. 1.5 months, p 0.001) but OS was similar (8.6 versus 6.9 months, $\mathrm{p}$ 0.48). Authors demonstrated that cetuximab is an active agent in mCRC specially in chemorefractory patients [39].

In 2007 it was published a trial which used cetuximab in chemorefractory patients whose tumors expressed EGFR by immunohistochemistry. When compared with best supportive care, patients who received cetuximab had a better OS (6.1 versus 4.6 months; HR of 0.77; CI, 0.64-0.92; p 0.005) [40]

Mutations in KRAS are present in $40 \%$ of patients and predict poorer outcome and resistance to Cetuximab or Panitumumab although a wt KRAS does not ensure response to these treatments. On the other hand, activating mutations of $B R A F$, mutually exclusive with mutations in $K-R A S$, occur in about $10 \%$ of patients and are also a poor prognostic factor. The NCCN guidelines recommends routine $K-R A S$ testing at the time of diagnosis of $\mathrm{mCRC}$ to facilitate the planning of treatment [43].

The CRYSTAL trial evaluated FOLFIRI plus or minus cetuximab in the first-line setting. It included 1217 patients. The results showed 
a statistically significant improvement in PFS ( 8.9 versus 8 months; $p$ 0.036)., response rate ( $46.9 \%$ versus $38.7 \%$, p 0.005 ), but with increased toxicity (specifically diarrhea and rash).

There was a better response rate and survival advantage for patients with KRAS wild-type tumors in the cetuximab arm (HR 0.796, p 0.0093). OS was 23.5 months in KRAS wild-type (wt) versus 16.2 in KRAS mutated (mt) [41,42].

The OPUS phase II trial studied FOLFOX with or without cetuximab as first-line. Its results reported a higher overall response rate favoring the cetuximab arm (45.6\% versus $35.7 \%)$. PFS was not statistically superior in the intention-to-treat arm, but it was better in the KRAS wild-type subgroup analysis [44].

A meta-analysis of both trials presented at ASCO 2010 confirmed the benefit in wt K-RAS of adding cetuximab: median OS was extended by 4 months ( 23.5 vs 19.5 , HR, 0.81, p 0.0062). The most common adverse effects of cetuximab are weakness, an acneiform rash, nausea, infusion-related reactions, and hypomagnesaemia. A relation among development, severity of treatment-induced skin rash, and response to anti-EGFR monoclonal antibodies has been suggested [45].

The BOND trial evaluated [46] patients with mCRC refractory to irinotecan (100\%) and oxaliplatin (63\%) with single-agent cetuximab or combination cetuximab and irinotecan. The combination achieved a better response rate $(22.9 \%$ versus $10.8 \%)$ with median time to progression of 4.1 and 1.5 months, respectively.

The study carried by National Cancer Institute of Canada evaluated cetuximab versus best supportive care in unselected patients with chemorefractory (fluoropyrimidine, oxaliplatin, irinotecan) mCRC [40]. The combination improved significantly PFS and OS.

Panitumumab demonstrated similar anti-tumor activity as a singleagent in patients with chemotherapy-refractory mCRC [47]. It showed an overall response rate of $10 \%$ and a very modest improvement in PFS compared with best supportive care (8 versus 7.3 weeks).

PRIME first-line trial (FOLFOX plus panitumumab) showed a statistically significant improvement in PFS (9.6 versus 8.0 months ( $\mathrm{HR}=0.80, \mathrm{p} 0.02)$ in wt KRAS tumors. A trend towards an OS benefit although did not reach significance was also seen. In patients with $\mathrm{mt}$ KRAS tumours, outcomes were inferior with panitumumab [48].

On the contrary the MRC COIN (CapeOx or FOLFOX and cetuximab) was a phase III trial which reported no significant differences in PFS and OS when anti-EGFR antibodies were added to first-line therapy in KRAS wt patients [49]. The authors found that for patients with KRAS wild-type tumours treated with infused fluorouracil, the benefit in PFS (HR for fluorouracil-based therapy was $0.77, p=0.06$, compared with HR for capecitabine-based therapy of $1.06, p=0.56, p$ for interaction 0.07 ) was consistent with other trials, concluding that this finding suggests the potential relevance of the agents used in combination when using EGFR-targeted therapies.

Further studies are needed to evaluate the impact of cetuximab in the survival of patients with wild-type $K$-RAS.

The phase III trial PACCE assigned patients to FOLFOXbevacizumab or FOLFIRI-bevacizumab, and then randomized them to either receive or not receive panitumumab [50].
This study showed a shorter PFS in the panitumumab arm. The CAIRO-2 trial [51] confirmed the results of the PACCE trial. It demonstrated that CapeOx-bevacizumab plus cetuximab got inferior response rates and PFS than CapeOx-bevacizumab alone.

The results provided by PACCE and CAIRO-2 trials indicated that combination of bevacizumab and EGFR inhibitory monoclonal antibody cannot be recommended in the front-line metastatic CRC as a standard treatment.

Globally no data are available comparing panitumumab and cetuximab (or either versus a bevacizumab-containing regimen), and the place of panitumumab, particularly for first-line therapy of mCRC, remains uncertain [35].

\section{Conclusions}

The choice of therapy for patients diagnosed with mCRC should be individually decided after careful consideration about the type and timing of previous chemotherapies, the goal of treatment, toxicity profiles, performance status and patient comorbidities.

The question we should answer before planning a treatment should be: Does the patient need an aggressive approach? If the answer is "yes", which occurs in $85 \%$ of patients, we should know KRAS status before the decision.

With mt or unavailable KRAS we could administer a chemotherapy doublet plus bevacizumab and with wt KRAS doublet plus cetuximab or bevacizumab.

In contrast if the answer is "no" fluoropyrimidines alone or in combination with bevacizumab would be acceptable options.

In selected patients we could use stop and maintenance treatments.

As a final conclusion there are still several controversial points which should be addressed in well design clinical trials. We hope to have more answers in the near future.

\section{References}

1. Jemal A, Siegel R, Xu J, Ward E (2010) Cancer statistics, 2010. CA Cancer J Clin 60: 277-300.

2. Altekruse SF (2007) SEER cancer statistics review, 1975-2007, Nationa Cancer Institute, Bethesda, MD

3. [No authors listed] (1998) Efficacy of intravenous continuous infusion of fluorouracil compared with bolus administration in advanced colorectal cancer. Meta-analysis Group in Cancer. J Clin Oncol 16: 301-308.

4. Thirion P, Michiels S, Pignon JP, Buyse M, Braud AC, et al. (2004) Modulation of fluorouracil by leucovorin in patients with advanced colorectal cancer: an updated meta-analysis. J Clin Oncol 22: 3766-3775.

5. Saltz LB, Clarke S, Díaz-Rubio E, Scheithauer W, Figer A, et al. (2008) Bevacizumab in combination with oxaliplatin-based chemotherapy as first-line therapy in metastatic colorectal cancer: a randomized phase III study. J Clin Oncol 26: 2013-2019.

6. Clinical Practice Guidelines in Oncology. September 10, 2010.

7. Scheithauer W, Rosen H, Kornek GV, Sebesta C, Depisch D (1993) Randomised comparison of combination chemotherapy plus supportive care with supportive care alone in patients with metastatic colorectal cancer. BM 306: $752-755$.

8. Nordic Gastrointestinal Tumor Adjuvant Therapy Group (1992) Expectancy or primary chemotherapy in patients with advanced asymptomatic colorectal cancer: a randomised trial. J Clin Oncol 10: 904-911. 
Citation: Cidón EU (2011) What is the Optimal Treatment for Metastatic Colorectal Cancer? Controversial Points. J Cancer Sci Ther S4:003. doi:10.4172/1948-5956.S4-003

Page 6 of 7

9. Ackland SP, Jones M, Tu D, Simes J, Yuen J, et al. (2005) A meta-analyses of two randomised trials of early chemotherapy in asymptomatic metastatic colorectal cancer. Br J Cancer 93: 1236-1243.

10. Miwa M, Ura M, Nishida M, Sawada N, Ishikawa T, et al. (1998) Design of a novel oral fluoropyrimidine carbamate, capecitabine, which generates 5 -fluorouracil selectively in tumours by enzymes concentrated in human liver and cancer tissue. Eur J Cancer 34: 1274-1281.

11. Schüller J, Cassidy J, Dumont E, Roos B, Durston S, et al. (2000) Preferential activation of capecitabine in tumor following oral administration to colorectal cancer patients. Cancer Chemother Pharmacol 45: 291-297.

12. Van Cutsem E, Twelves C, Cassidy J, Allman D, Bajetta E, et al. (2001) Oral capecitabine compared with intravenous fluorouracil plus leucovorin in patients with metastatic colorectal cancer: results of a large phase III study. J Clin Oncol 19: $4097-4106$

13. Hoff PM, Ansari R, Batist G, Cox J, Kocha W, et al. (2001) Comparison of oral capecitabine versus intravenous fluorouracil plus leucovorin as firstline treatment in 605 patients with metastatic colorectal cancer: results of a randomized phase III study. J Clin Oncol 19: 2282-2292.

14. Díaz-Rubio E, Tabernero J, Gómez-España A, Massutí B, Sastre J, et al. (2007) Phase III study of capecitabine plus oxaliplatin compared with continuousinfusion fluorouracil plus oxaliplatin as first-line therapy in metastatic colorectal cancer: final report of the Spanish Cooperative Group for the Treatment of Digestive Tumors Trial. J Clin Oncol 25: 4224-4230.

15. Porschen R, Arkenau HT, Kubicka S, Greil R, Seufferlein T, et al. (2007) Phase III study of capecitabine plus oxaliplatin compared with fluorouracil and leucovorin plus oxaliplatin in metastatic colorectal cancer: a final report of the AIO Colorectal Study Group. J Clin Oncol 25: 4217-4223.

16. Cassidy J, Clarke S, Díaz-Rubio E, Scheithauer W, Figer A, et al. (2008) Randomized phase III study of capecitabine plus oxaliplatin compared with fluorouracil/folinic acid plus oxaliplatin as first-line therapy for metastatic colorectal cancer. J Clin Oncol 26: 2006-2012.

17. Sakamoto J, Ohashi Y, Hamada C, Buyse M, Burzykowski T, et al. (2004) Efficacy of oral adjuvant therapy after resection of colorectal cancer: 5-year results from three randomized trials. J Clin Oncol 22: 484-492.

18. Köhne CH, De Greve J, Hartmann JT, Lang I, Vergauwe P, et al. (2008) Irinotecan combined with infusional 5-fluorouracil/folinic acid or capecitabine plus celecoxib or placebo in the first-line treatment of patients with metastatic colorectal cancer. EORTC study 40015. Ann Oncol 19: 920-926.

19. Fuchs CS, Marshall J, Mitchell E, Wierzbicki R, Ganju V, et al. (2007) Randomized, controlled trial of irinotecan plus infusional, bolus, or oral fluoropyrimidines in first-line treatment of metastatic colorectal cancer: results from the BICC-C study. J Clin Oncol 25: 4779-4786.

20. Haller DG, Cassidy J, Clarke SJ, Cunningham D, Van Cutsem E, et al. (2008) Potential regional differences for the tolerability profiles of fluoropyrimidines. J Clin Oncol 26: 2118-2123.

21. Koopman M, Antonini NF, Douma J, Wals J, Honkoop AH, et al. (2007) Sequential versus combination chemotherapy with capecitabine, irinotecan, and oxaliplatin in advanced colorectal cancer (CAIRO): a phase III randomised controlled trial. Lancet 370: 135-142.

22. Seymour MT, Maughan TS, Ledermann JA, Topham C, James R, et al. (2007) Different strategies of sequential and combination chemotherapy for patients with poor prognosis advanced colorectal cancer (MRC FOCUS): a randomised controlled trial. Lancet 370: 143-152.

23. Grothey A, Sargent D, Goldberg R, Schmoll HJ (2004) Survival with advanced colorectal cancer improves with the availability of fluorouracil-leucovorin, irinotecan and oxaliplatin in the course of treatment. J Clin Oncol 22: 12091214.

24. Chibaudel B, Maindrault-Goebel F, Lledo G, Mineur L, André T, et al. (2009) Can chemotherapy be discontinued in unresectable metastatic colorectal cancer? The GERCOR OPTIMOX2 study. J Clin Oncol 27: 5727-5733.

25. Adams RA, Meade AM, Seymour MT, Wilson RH, Madi A, et al. (2011) Intermittent versus continuous oxaliplatin and fluoropyrimidine combination chemotherapy for first-line treatment of advanced colorectal cancer: results of the randomised phase 3 MRC COIN trial. Lancet Oncol 12: 642-653.

26. Labianca R, Sobrero A, Isa L, Cortesi E, Barni S, et al. (2006) Alternating versus continuous "FOLFIRI" in advanced colorectal cancer (ACC): A randomized "GISCAD" trial. Ann Oncol 22: 1236-1242.

27. J Tabernero, E Aranda, A Gomez, B Massuti (2010) Phase III study of first-line XELOX plus bevacizumab (BEV) for 6 cycles followed by XELOX plus BEV or single-agent (s/a) BEV as maintenance therapy in patients (pts) with metastatic colorectal cancer $(\mathrm{mCRC})$ : The MACRO Trial (Spanish Cooperative Group for the Treatment of Digestive Tumors [TTD]). J Clin Oncol 28: 15s.

28. Grothey A, Hart LL, Rowland KM, Ansari RH (2008) Intermittent oxaliplatin administration and time-to-treatment failure (TTF) in metastatic colorecta cancer (mCRC): Final results of the phase III CONcePT trial. J Clin Oncol 26: $15 \mathrm{~S}$

29. Tournigand C, Cervantes A, Figer A, Lledo G, Flesh M, et al. (2006) OPTIMOX1 A randomized study of FOLFOX4 or FOLFOX7 with oxaliplatin in a stop-andGo fashion in advanced colorectal cancer-a GERCOR study. J Clin Oncol 24: 394-400.

30. Tournigand C, Andre T, Achille E, Lledo G, Flesh M, et al. (2004) FOLFIRI followed by FOLFOX6 or the reverse sequence in advanced colorectal cancer: a randomized GERCOR study. J Clin Oncol 22: 229-237.

31. Avastin product information.

32. Hurwitz H, Fehrenbacher L, Novotny W, Cartwright T, Hainsworth J, et al (2004) Bevacizumab plus irinotecan, fluorouracil, and leucovorin for metastatic colorectal cancer. N Engl J Med 350: 2335-2342.

33. Saltz LB, Clarke S, Díaz-Rubio E, Scheithauer W, Figer A, et al. (2008) Bevacizumab in combination with oxaliplatin-based chemotherapy as first-line therapy in metastatic colorectal cancer: a randomized phase III study. J Clin Oncol 26: 2013-2019.

34. Giantonio BJ, Catalano PJ, Meropol NJ, O'Dwyer PJ, Mitchell EP, et al. (2007) Bevacizumab in combination with oxaliplatin, fluorouracil, and leucovorin (folfox4) for previously treated metastatic colorectal cancer: results from the eastern cooperative oncology group study e3200. J Clin Oncol 25: 1539-1544.

35. Grothey A, Sugrue MM, Purdie DM, Dong W, Sargent D, et al. (2008) Bevacizumab beyond first progression is associated with prolongued overal survival in metastatic colorectal cancer: results from a large observational cohort study (BRiTE). J Clin Oncol 26: 5326-5334.

36. http://www.clinicaltrials.gov/ct/show/NCT00499369.

37. http://webres.uccrc.org/clinical_trials.

38. http://clinicaltrials.gov/ct2/show/NCT00418938.

39. Cunningham D, Humblet $Y$, Siena S, Khayat D, Bleiberg H, et al. (2004) Cetuximab monotherapy and cetuximab plus irinotecan in irinotecan-refractory metastatic colorectal cancer. N Engl J Med 351: 337-345.

40. Jonker DJ, O'Callaghan CJ, Karapetis CS, Zalcberg JR, Tu D, et al. (2007) Cetuximab for the treatment of colorectal cancer. N Engl J Med 357: 2040 2048.

41. Van Cutsem E, Köhne CH, Hitre E, Zaluski J, Chang Chien CR, et al. (2009) Cetuximab and Chemotherapy as initial treatment for metastatic colorectal cancer. N Engl J Med 360: 1408-1417.

42. Van Cutsem E, Köhne CH, Láng I, Folprecht G, Nowacki MP, et al. (2011) Cetuximab plus irinotecan, fluorouracil, and leucovorin as first-line treatment for metastatic colorectal cancer: updated analysis of overall survival according to tumour KRAS and BRAF mutation status. J Clin Oncol 29: 2011-2019.

43. Clinical Practice Guidelines in Oncology

44. Bokemeyer C, Bondarenko I, Makhson A, Hartmann JT, Aparicio J, et al. (2009) Fluorouracil, leucovorin, and oxaliplatin with and without cetuximab in the firstline treatment of metastatic colorectal cancer. J Clin Oncol 27: 663-671.

45. Bokemeyer C, Bondarenko I, Hartmann JT, de Braud F, Schuch G, et al. (2011) Efficacy according to biomarker status of cetuximab plus FOLFOX-4 as first 
Citation: Cidón EU (2011) What is the Optimal Treatment for Metastatic Colorectal Cancer? Controversial Points. J Cancer Sci Ther S4:003. doi:10.4172/1948-5956.S4-003

line treatment for metastatic colorectal cancer: the OPUS study. Ann Oncol 22: $1535-1546$

46. Saltz LB, Lenz HJ, Kindler HL, Hochster HS, Wadler S, et al. (2007) Randomized phase II trial of cetuximab, bevacizumab, and irinotecan compared with cetuximab and bevacizumab alone in irinotecan-refractory colorectal cancer: the BOND-2 study. J Clin Oncol 25: 4557-4561.

47. Van Cutsem E, Peeters M, Siena S, Humblet Y, Hendlisz A, et al. (2007) Openlabel phase III trial of panitumumab plus best supportive care compared with best supportive care alone in patients with chemotherapy-refractory metastatic colorectal cancer. J Clin Oncol 25:1658-1664.

48. Douillard JY, Siena S, Cassidy J, Tabernero J, Burkes R, et al. (2010) Randomized phase III study of panitumumab with infusional fluorouracil leucovorin and oxaliplatin (FOLFOX4) versus FOLFOX4 alone as first-line treatment in patients with previously untreated metastatic colorectal cancer: the PRIME study. J Clin Oncol 28: 4697-4705

49. Maughan TS, Adams RA, Smith CG, Meade AM, Seymour MT, et al. (2011) Addition of cetuximab to oxaliplatin-based first-line combination chemotherapy for treatment of advanced colorectal cancer: results of the randomised phase III MRC COIN trial. Lancet 377: 2103-2114.

50. Hecht JR, Mitchell E, Chidiac T, Scroggin C, Hagenstad C, et al. (2009) A randomized phase IIIB trial of chemotherapy, bevacizumab, and panitumumab compared with chemotherapy and bevacizumab alone for metastatic colorectal cancer. J Clin Oncol 27: 672-680.

51. Tol J, Koopman M, Cats A, Rodenburg CJ, Creemers GJ, et al. (2009) Chemotherapy, bevacizumab, and cetuximab in metastatic colorectal cancer N Engl J Med 360: 563-572. 